\title{
AMENDMENTS
}

\section{Author Correction: Enhanced nucleotide chemistry and toehold nanotechnology reveals IncRNA spreading on chromatin}

\author{
Martin Machyna (D), Lea Kiefer and Matthew D. Simon (D)
}

Correction to: Nature Structural \& Molecular Biology https://doi.org/10.1038/s41594-020-0390-z, published online 10 March 2020.

In the version of this article initially published, in the caption for Extended Data Fig. 4i, Extended Data Fig. $1 \mathrm{j}$ was incorrectly cited as "Supplementary fig. 1j". The error has been corrected in the HTML and PDF versions of the article.

In the supplementary information originally posted for this article, Supplementary Table 1 did not accurately reflect the oligonucleotide sequences used in this study. The last three sequences in the table, "polyA LNA CO", "polyA PO" and "polyA EO" were not used in this study. These have been replaced with the sequences that were used: "TERC LNA CO", TERC PO" and "TERC EO". The errors have been corrected online.

Published online: 18 March 2020

https://doi.org/10.1038/s41594-020-0413-9

๑) The Author(s), under exclusive licence to Springer Nature America, Inc. 2020

\section{Author Correction: An allosteric network in spastin couples multiple activities required for microtubule severing}

\author{
Colby R. Sandate (iD, Agnieszka Szyk, Elena A. Zehr, Gabriel C. Lander (D) and Antonina Roll-Mecak (D)
}

Correction to: Nature Structural \& Molecular Biology https://doi.org/10.1038/s41594-019-0257-3, published online 8 July 2019.

In the supplementary information originally posted for this article, Supplementary Table 1 was missing. The table is now available online.

Published online: 19 March 2020

https://doi.org/10.1038/s41594-020-0414-8

$\odot$ The Author(s), under exclusive licence to Springer Nature America, Inc. 2020

\section{Publisher Correction: Global site-specific neddylation profiling reveals that NEDDylated cofilin regulates actin dynamics}

Annette M. Vogl D, Lilian Phu, Raquel Becerra, Sebastian A. Giusti, Erik Verschueren, Trent B. Hinkle,

Martín D. Bordenave, Max Adrian, Amy Heidersbach, Patricio Yankilevich, Fernando D. Stefani, Wolfgang Wurst,

Casper C. Hoogenraad, Donald S. Kirkpatrick (D), Damian Refojo (D) and Morgan Sheng (D)

Correction to: Nature Structural \& Molecular Biology https://doi.org/10.1038/s41594-019-0370-3, published online 3 February 2020.

In the version of this article initially published online, in Fig. 6d, the third and fourth bars were incorrectly labeled "DMSO + cytochrome D" and "MLN4924 + cytochrome D," respectively. They should have been labeled "DMSO + cytochalasin D" and "MLN4924 + cytochalasin D," respectively. The errors have been corrected in the print, PDF and HTML versions of the article.

Published online: 26 February 2020

https://doi.org/10.1038/s41594-020-0395-7

(c) The Author(s), under exclusive licence to Springer Nature America, Inc. 2020 